\title{
FERMION DETERMINANTS
}

\author{
M. P. FRY \\ School of Mathematics, University of Dublin \\ Dublin 2, Ireland
}

\begin{abstract}
The current status of bounds on and limits of fermion determinants in two, three and four dimensions in QED and QCD is reviewed. A new lower bound on the two-dimensional QED determinant is derived. An outline of the demonstration of the continuity of this determinant at zero mass when the background magnetic field flux is zero is also given.
\end{abstract}

\section{Introduction}

The calculation of fermion determinants is an old problem that has reemerged as part of mainstream physics. They lie at the heart of gauge field theories with fermions and appear in the calculation of every physical process. Their increasing interest is due to lattice QCD and the improvement of lattice fermion actions. They are obtained by integrating over the fermion fields analytically to produce the one-loop effective action $S_{\text {eff }}=-\ln$ det, where det is formally the ratio $\operatorname{det}(\not P-e A+m) / \operatorname{det}(\not P+m)$ of Fredholm determinants of euclidean Dirac operators. Properly defined, det is a gauge invariant but nonlocal function of the background gauge potential $A_{\mu}$, and it is this nonlocality that makes det so challenging to calculate both analytically and numerically. At present the best that can be hoped for from analytic nonperturbative calculations are restrictive upper and lower bounds on determinants in two, three and four dimensions, as well as particular limits, such as strong coupling or small fermion mass. Such results are relevant to lattice calculations extrapolated to the continuum as they are a nontrivial test of lattice discretization procedures, algorithms, and practices such as taking the square root of the Kogut-Susskind determinant to simulate two degenerate quark flavors.

In Sec. 2 we report on the current status of bounds on and limits of fermion determinants. In Sec. 3 the derivation of a new lower bound on the two-dimensional QED determinant is given. Section 1 contains the proof that the chiral limit of this determinant coincides with the Schwinger model's determinant only when the background magnetic field's flux is zero.

\section{Bounds and Limits on det}

In order to make estimates the class of background gauge fields has to be defined. Since the determinant is part of the gauge field's action, $A_{\mu}$ and the field strength $F_{\mu \nu}$ are random fields. However, there is a need to regulate in any dimension. 
For $A_{\mu} \in S^{\prime}$, the Schwartz space of tempered distributions, this can be done by smoothing $A_{\mu}$ in the determinant and elsewhere, except in the gauge-fixed Gaussian measure $d \mu(A)$ for $A_{\mu}$, by convoluting it with a function $h_{\Lambda} \in S$, the functions of rapid decrease. This gives a potential $A_{\mu}^{\Lambda}(x)=\left(h_{\Lambda} * A_{\mu}\right)(x)$ that is a polynomial bounded $C^{\infty}$ function whose covariance is now

$$
\int d \mu(A) A_{\mu}^{\Lambda}(x) A_{\nu}^{\Lambda}(y)=D_{\mu \nu}^{\Lambda}(x-y) .
$$

The Fourier transform of $D_{\mu \nu}^{\Lambda}$ is proportional to $\left|\hat{h}_{\Lambda}\right|^{2}$, where $\hat{h}_{\Lambda}$ is the Fourier transform of $h_{\Lambda}$ and has the property that $\hat{h}_{\Lambda}=0$ for momenta $k^{2}>\Lambda^{2}$. Therefore without loss of generality we may assume smooth potentials and fields. In addition they must be in some $L^{n}\left(\mathbb{R}^{d}\right)$ space(s) that will be stated explicitly below.

All results, except the upper bounds for $d \overline{\overline{\mathrm{B}}} 2,3$, are for an abelian field obtained by using Schwinger's proper time definitiona of the determinant as the starting point, namely

$$
\begin{aligned}
\text { ln det } & =\frac{1}{2} \int_{0}^{\infty} \frac{d t}{t} \\
& \left(\operatorname{Tr}\left\{e^{-P^{2} t}-\exp \left[-\left((D-e A)^{2}+\frac{1}{2} e \sigma^{\mu \nu} F_{\mu \nu}\right) t\right]\right\}+\frac{e^{2}\|F\|^{2}}{24 \pi^{2}}\right) e^{-t m^{2}},
\end{aligned}
$$

where $\sigma^{\mu \nu}=\left[\gamma^{\mu}, \gamma^{\nu}\right] / 2 i, \gamma^{\mu \dagger}=-\gamma^{\mu}$, and $\|F\|^{2}=\int d^{4} x F_{\mu \nu}^{2}(x)$. The last term is the second-order charge renormalization subtraction at zero momentum transfer required for the integral to converge for small $t$ in $d=4$. A local counterterm is not required to define lndet in $d<4$, and so the last term in (2) will be omitted in this case.

$\mathbf{d}=\mathbf{2}$

The calculation of (2) in this case requires knowledge of the bound and scattering states of a charged fermion confined to a plane in the presence of a static magnetic field $\mathrm{B}$ perpendicular to the plane. Of course there is actually no third dimension in this strictly two dimensional problem. For $A_{\mu} \in \bigcap_{n>2} L^{n}\left(\mathbb{R}^{2}\right)$,

$$
--\frac{\|e B\|^{2}}{4 \pi m^{2}} \leq \ln \operatorname{det} \leq 0 .
$$

In addition, the lower bound requires that $B$ be square-integrable. This bound will be derived in Sec. 3. A sharper lower bound for a unidirectional magnetic field $B(x) \geq 0$ is given by

$$
\ln \operatorname{det} \geq \frac{1}{4 \pi} \int d^{2} x\left[e B(x)-\left[m^{2}+e B(x)\right] \ln \left(1+\frac{e B(x)}{m^{2}}\right)\right] .
$$


The upper bound in (3) was obtained as a limit国 of lattice estimates.6. It is referred to as the "diamagnetic" bound though physically it is a reflection of the paramagnetism of a charged fermion in an external magnetic field as (2) makes clear. Moreover, the upper bound also holds for nonabelian fields. 6 t There is the technical problem of proving that the lattice limit coincides with the renormalized determinant $d^{2} t_{r e n}$ defined in Refs. $0,5,8$ and that $\operatorname{det}_{r e n}$ can in turn be identified with definition (2). This is dealt with in Refs. 4. It would be desirable to have a continuum proof of the upper bound in (3).

If in addition $B$ has a finite range then, for $m^{2} \rightarrow 0$

$$
\ln \operatorname{det}=\frac{|e \Phi|}{4 \pi} \ln m^{2}+R\left(m^{2}\right),
$$

where $\lim _{m \rightarrow 0}\left[R\left(m^{2}\right) / \ln m^{2}\right]=0$ and $\Phi=\int d^{2} x B(x)$. 9 For integer values of $e \Phi / 2 \pi$ (5) is intuitively expected by the Aharonov-Casher theorem 10 which states that the number of zero modes of $\not P-e A$ is $[|e \Phi| / 2 \pi]$, all with positive or negative chirality, where $[x]$ stands for the nearest integer less than $x$ and $[0]=0$. This result indicates that the zero-mass limit of det does not coincide with the Schwinger model except when $\Phi=0$. The proof of continuity at $m=0$ will be outlined in Sec. 1 .

As a corollary of (5) suppose we define $l n \operatorname{det}_{3}$ as

$$
\text { ln } \operatorname{det}=-\frac{e^{2}}{2 \pi} \int \frac{d^{2} k}{(2 \pi)^{2}}|\hat{B}(k)|^{2} \int_{0}^{1} d z \frac{z(1-z)}{k^{2} z(1-z)+m^{2}}+\ln \operatorname{det}_{3},
$$

where the first term is just second-order perturbation theory and $\ln \operatorname{det}_{3}$ is the remainder. Then provided $0<|e \Phi|<2 \pi$,

there is at least one value of $m^{2}>0$ for which $l n \operatorname{det}_{3}=0.11$ This means the following: it is known that $\operatorname{lndet}_{3}\left(m^{2}=0\right)=0$ as first shown by Schwinger.12 Continuity of $l n \operatorname{det}_{3}$ at $m^{2}=0$ when $\Phi=0$ implies $\lim _{m^{2}=0} l n \operatorname{det}_{3}=0$. Equations (5) and (6) imply that lndet $_{3}<0$ if $0<|e \Phi|<2 \pi$ and $m^{2}$ is sufficiently small, while it must become positive before approaching zero as $m^{2} \rightarrow \infty .11$ So our result states that when $0<|e \Phi|<2 \pi$, the zero in $\ln _{\text {det }}$ moves from $m=0$ when $\Phi=0$ to some finite value(s) when $m^{2}>0$. No information is available yet on mass zero(s) when $|e \Phi| \geq 2 \pi$.

$\mathrm{d}=3$

Here we have

$$
--\frac{Z}{6 \pi} \int d^{2} x|e B(x)|^{\frac{3}{2}} \leq \ln \operatorname{det} \leq 0 .
$$

As for $d=2$ the upper bound was obtained as a limit $\mathrm{Qt}_{\mathrm{O}}$ of lattice estimates 6 and holds also for nonabelian fields provided $A_{\mu} \in \bigcap_{n>3} L^{n}\left(\mathbb{R}^{3}\right)$. This restriction on $A_{\mu}$ is sufficient to make mathematical sense out of the renormalized determinant 
$\operatorname{det}_{r e n}$ referred to above. The same technical problem of limits referred to for $d=2$ persists here.

The lower bound 33 is valid only for unidirectional fields $B(x) \geq 0$ with $x \in \mathbb{R}^{2}$; hence the box cutoff $Z$ in the direction of $B$. In addition, the derivation assumed that $A_{\mu} \in \bigcap_{n>2} L^{n}\left(\mathbb{R}^{2}\right)$, that $B$ has finite flux and $B \in L^{n}\left(\mathbb{R}^{2}\right), n=2, \frac{3}{2}$. A $2 \mathrm{x} 2$ representation of the Dirac matrices was used. The definition (and regularization) of the fermion determinant in (2) is parity conserving and gives no Chern-Simons term, which is known to be regularization dependent 14 The analysis leading to the upper bound in (7) predated the discovery of the Chern-Simons term and is not included in the definition of det.

Another lower bound may be obtained from the lower bound in (3) and the connection between the $\mathrm{QED}_{2}$ and $\mathrm{QED}_{3}$ determinants, 13 namely

$$
\text { ln } \operatorname{det}_{\mathrm{QED} 3}=\frac{Z}{2 \pi} \int_{m^{2}}^{\infty} \frac{d M^{2}}{\sqrt{M^{2}-m^{2}}} \ln \operatorname{det}_{\mathrm{QED} 2}\left(M^{2}\right) .
$$

Eqs. (3) and (8) immediately give

$$
\ln \operatorname{det}_{\mathrm{QED} 3} \geq-\frac{Z \|\left. e B\right|^{2}}{16 \pi|m|},
$$

where in this bound $B$ no longer has to be unidirectional.

$\mathrm{d}=4$

In this case only strong coupling limits are known. Thus if $e \rightarrow \lambda e$, then

$$
\frac{\|e B\|^{2} Z T}{48 \pi^{2}} \leq \lim _{\lambda \rightarrow \infty}\left(\frac{\ln \operatorname{det}}{\lambda^{2} \ln \lambda}\right) \leq \frac{\|e B\|^{2} T}{6 \pi^{2}} .
$$

The upper bound 13 requires $A \in L^{3}\left(\mathbb{R}^{3}\right)$ and $B \in L^{2}\left(\mathbb{R}^{3}\right)$. If $\mathbf{A}$ is also in the Coulomb gauge $\nabla \cdot \mathbf{A}=0$ then we showed 13 that $A_{\mu} \in \bigcap_{3 \leq n \leq 6} L^{n}\left(\mathbb{R}^{3}\right)$, which guarantees that ln det in (10) is well-defined. Then the upper bound in (10) is valid for general square-integrable static magnetic fields; $T$ is the (euclidean) time cutoff. The main input to this bound is the upper bound on $l n$ det in $d=3$ given by (7). The lower bound 3 holds only for square-integrable magnetic fields $B(x) \geq 0$ and is derived from the lower bound (价).

Finally we note that it is possible to make a derivative expansion of $l n$ det into a sum of terms containing increasing numbers of derivatives of $F_{\mu \nu} .15$ Such an approach has the merit of going nonperturbatively beyond the constant field approximation. Nevertheless, it is an expansion, and either all terms are summed or the series is terminated and the remainder is bounded. Investigation of this problem has begun with some exactly solvable cases 10 For the special case of a $\operatorname{sech}^{2}(x / \lambda)$ magnetic field it is found that the derivative expansion of ln det is a divergent asymptotic series that is Borel summable.16 This is encouraging. As far 
as bounds on ln det are concerned, all that is required is to show that the series is asymptotic in the strict mathematical sense because then one has a bound on the remainder of the series after any number of terms.

\section{Lower Bound on ln det in $d=2$}

The lower bound in (3) was originally derived for a unidirectional field $B \geq 0$ in Ref. 3. We have since noticed that it is easy to generalize this result to a general square-integrable field. From here on the coupling constant e is absorbed into the potential: $e A_{\mu} \rightarrow A_{\mu}$.

From definition (2), without the charge renormalization term,

$$
\frac{\partial}{\partial m^{2}} \operatorname{lndet}=\frac{1}{2} \operatorname{Tr}\left[\left(D^{2}-\sigma_{3} B+m^{2}\right)^{-1}-\left(P^{2}+m^{2}\right)^{-1}\right],
$$

where $D=P-A$. Using

$$
\begin{aligned}
& \left(D^{2}-\sigma_{3} B+m^{2}\right)^{-1}=\left(D^{2}+m^{2}\right)^{-1}+\left(D^{2}+m^{2}\right)^{-1} \sigma_{3} B\left(D^{2}+m^{2}\right)^{-1} \\
& +\left(D^{2}+m^{2}\right)^{-1} \sigma_{3} B\left(D^{2}+m^{2}\right)^{-1} \sigma_{3} B\left(D^{2}-\sigma_{3} B+m^{2}\right)^{-1},
\end{aligned}
$$

(11) becomes

$$
\begin{aligned}
\frac{\partial}{\partial m^{2}} \operatorname{lndet}=\frac{1}{2} \operatorname{Tr}\left[\left(D^{2}+m^{2}\right)^{-1}-\left(P^{2}+m^{2}\right)^{-1}\right. \\
\left.+\left(D^{2}+m^{2}\right)^{-1} \sigma_{3} B\left(D^{2}+m^{2}\right)^{-1} \sigma_{3} B\left(D^{2}-\sigma_{3} B+m^{2}\right)^{-1}\right] .
\end{aligned}
$$

Because of the definition (2) the trace of the first two terms in (13) is defined by the left-hand side of

$$
\int_{0}^{\infty} d t \operatorname{Tr}\left[e^{-(P-A)^{2} t}-e^{-P^{2} t}\right] e^{-t m^{2}} \leq 0 ;
$$

the right-hand side follows from Kato's inequality in the form stated by the authors in Ref. 17. Hence,

$$
\frac{\partial}{\partial m^{2}} \operatorname{lndet} \leq \frac{1}{2} \operatorname{Tr}\left[\left(D^{2}+m^{2}\right)^{-1} \sigma_{3} B\left(D^{2}+m^{2}\right)^{-1} \sigma_{3} B\left(D^{2}-\sigma_{3} B+m^{2}\right)^{-1}\right] .
$$

By the Schwarz inequality, $|\operatorname{Tr}(A B)| \leq\|A\|_{2}\|B\|_{2}$,

$$
\left|\operatorname{Tr}\left[\left(D^{2}+m^{2}\right)^{-1} \sigma_{3} B\left(D^{2}+m^{2}\right)^{-1} \sigma_{3} B\left(D^{2}-\sigma_{3} B+m^{2}\right)^{-1}\right]\right| \leq\|\alpha\|_{2}\|\beta\|_{2},
$$

where

$$
\begin{aligned}
\alpha & =\left(D^{2}+m^{2}\right)^{-1} \sigma_{3} B \\
\beta & =\left(D^{2}+m^{2}\right)^{-1} \sigma_{3} B\left(D^{2}-\sigma_{3} B+m^{2}\right)^{-1} .
\end{aligned}
$$


Setting $<x\left|\left(D^{2}+m^{2}\right)^{-1}\right| y>=\Delta(x, y \mid A)$,

$$
\begin{aligned}
\|\alpha\|_{2}^{2} & =\operatorname{Tr}\left(\sigma_{3} B \Delta^{\dagger}(A) \Delta(A) \sigma_{3} B\right) \\
& \leq \operatorname{Tr}\left(B \Delta^{2}(A=0) B\right) \\
& =\frac{\|B\|^{2}}{2 \pi m^{2}}
\end{aligned}
$$

where use was made of the diamagnetic (Kato's) inequality

$$
|\Delta(x, y \mid A)| \leq \Delta(x-y),
$$

in the form given in Ref. 18.

Next,

$$
\begin{aligned}
\|\beta\|_{2} & \leq\left\|\left(D^{2}-\sigma_{3} B+m^{2}\right)^{-1}\right\|_{\infty}\left\|\left(D^{2}+m^{2}\right)^{-1} \sigma_{3} B\right\|_{2} \\
& =\frac{\|\alpha\|_{2}}{m^{2}},
\end{aligned}
$$

since $D^{2}-\sigma_{3} B \geq 0$.

Combining (15), (16), (18), and (20) gives

$$
\frac{\partial}{\partial m^{2}} \operatorname{lndet} \leq \frac{\|B\|^{2}}{4 \pi m^{4}} .
$$

Now integrate (21) from $m^{2}$ to $m^{2}=\infty$ and set $\operatorname{det}\left(m^{2}=\infty\right)=1$. This does not conflict with any renormalization condition. It is physically reasonable since an infinite-mass fermion cannot respond to an external magnetic field. Then

$$
\operatorname{lndet} \geq-\frac{\|B\|^{2}}{4 \pi m^{2}},
$$

which is the left-hand side of (3).

\section{Continuity of lndet in $d=2$ at $m=0$ when $\Phi=0$}

From (6) we want to show that

$$
\begin{aligned}
\lim _{m=0} \operatorname{lndet} & =\operatorname{lndet}_{\text {Schwinger model }} \\
& =\frac{1}{4 \pi^{2}} \int d^{2} x d^{2} y B(x) B(y) \ln |x-y|,
\end{aligned}
$$

and in particular that

$$
\lim _{m=0} \operatorname{lndet}_{3}=0
$$


if $\Phi=0$ and $A_{\mu} \in \bigcap_{n>1} L^{n}\left(\mathbb{R}^{2}\right)$. The analysis is simplified if $B$ has finite range, which we will assume. Then the limit in Eq. (23) comes from the first term in Eq. (6). The demonstration of (24) is not in the literature to our knowledge. It is surprisingly tedious and only its outline will be given.

\section{Step 1.}

Let $S=(\not p+m)^{-1}$. Neither $S A$ nor $(S A)^{2}$ are trace class operators, while $(S A)^{3}$ is. Therefore the identity $\operatorname{lndet}(1+A)=\operatorname{Tr} \ln (1+A)$ for trace class operators has to be modified to the regularized Fredholm determinant 612

$$
\operatorname{lndet}_{3}(1-S A)=\operatorname{Tr}\left[\ln (1-S A)+S A+\frac{1}{2}(S A)^{2}\right] .
$$

The connection of $\operatorname{lndet}_{3}$ to definition (2) is given in Ref. 11. The effect of (25) is to remove the ambiguous second-order graph from the determinant; it is defined by the second-order expansion of (2), giving the first term in (6). The operator $S A$ is a compact operator on $L^{2}\left(\mathbb{R}^{2} \sqrt{k^{2}-m^{2}} d^{2} k ; \mathbb{C}^{2}\right)$ for $A_{\mu} \in \bigcap_{n>2} L^{n}\left(\mathbb{R}^{2}\right)$, $\left[\mathcal{C}_{n}=\left\{A \mid\|A\|_{n}^{n} \equiv \operatorname{Tr}\left(A^{\dagger} A\right)^{\frac{n}{2}}<\infty\right\}\right]$, 4 , 22 Because $S A \in \mathcal{C}_{3}$, one more loop (i.e one more order in $A$ ) can be subtracted from the determinant to give 20.21

$$
\operatorname{lndet}_{4}(1-S A)=\operatorname{lndet}_{3}(1-S A)+\frac{1}{3} \operatorname{Tr}(S A)^{3} .
$$

By C-invariance $\operatorname{Tr}(S A)^{3}=0$. At this point it is useful to make a similarity transformation 4 and consider $\operatorname{det}_{4}\left(1-K_{m}\right)$, where

$$
K_{m}=\frac{m-\not p}{\left(p^{2}+m^{2}\right)^{\frac{3}{4}}} A \frac{1}{\left(p^{2}+m^{2}\right)^{\frac{1}{4}}},
$$

is a compact operator on two-component square-integrable functions on $\mathbb{R}^{2}$.

\section{Step 2.}

The main theorem required to show that $\lim _{m=0} \operatorname{lndet}_{4}=0$ is Simon's theorem 6.5 specialized to $\operatorname{det}_{4}$ :

$$
\begin{aligned}
\left|\operatorname{det}_{4}\left(1-K_{m}\right)-\operatorname{det}_{4}\left(1-K_{0}\right)\right| & \\
& \leq\left\|K_{m}-K_{0}\right\|_{4} \exp \left[\Gamma_{4}\left(\left\|K_{m}\right\|_{4}+\left\|K_{0}\right\|_{4}+1\right)^{4}\right],
\end{aligned}
$$

where $\frac{1}{1} \leq \Gamma_{4} \leq \frac{3}{4} 20$ Here $K_{0}$ is $K_{m}$ with $m=0$, which is known to be in $\mathcal{C}_{n}$, $n>2.23$

Furthermore,23

$$
\operatorname{det}_{4}\left(1-K_{0}\right)=1
$$


It is essential for the proof of this that $A_{\mu}$ is "nonwinding" so that $A_{\mu} \in \bigcap_{n>1} L^{2}\left(\mathbb{R}^{2}\right)$. [This condition was not stated in Refs. 4, 23. But it is necessary, and the proofs leading to (29) still go through.] Therefore by (28), (29) and (26) the demonstration of (24) requires that $\lim _{m=0}\left\|K_{m}-K_{0}\right\|_{4}=0$.

\section{Step 3.}

The operator difference $K_{m}-K_{0}$ may be decomposed into a sum of five terms so that

$$
\begin{aligned}
& \left\|K_{m}-K_{0}\right\|_{4} \leq\left\|\frac{\not p}{|p|}\left(\frac{1}{|p|^{\frac{1}{2}}} A \frac{1}{\left(p^{2}+m^{2}\right)^{\frac{1}{4}}}-\frac{|p|}{\left(p^{2}+m^{2}\right)^{\frac{3}{4}}} A \frac{1}{\left(p^{2}+m^{2}\right)^{\frac{1}{4}}}\right)\right\|_{4} \\
& +\left\|\frac{\not p}{|p|} \frac{1}{|p|^{\frac{1}{2}}} A\left(\frac{1}{|p|^{\frac{1}{2}}}-\frac{1}{\left(p^{2}+m^{2}\right)^{\frac{1}{4}}}\right)\right\|_{4} \\
& +m\left\|\frac{1}{\left(p^{2}+m^{2}\right)^{\frac{3}{4}}} A \frac{1}{\left(p^{2}+m^{2}\right)^{\frac{1}{4}}}\right\|_{4} .
\end{aligned}
$$

The unitary $\not p /|p|$ is irrelevant to the norms and will be dropped from here on. We now outline the $m=0$ limit of the first norm.

By Hölder's inequality, $\|f g\|_{r} \leq\|f\|_{p}\|g\|_{q}$ for $p^{-1}+q^{-1}=r^{-1}$, and $p, q, r \geq 1$,

$$
\begin{aligned}
\left\|\frac{1}{|p|^{\frac{1}{2}}} A \frac{1}{\left(p^{2}+m^{2}\right)^{\frac{1}{4}}}-\frac{|p|}{\left(p^{2}+m^{2}\right)^{\frac{3}{4}}} A \frac{1}{\left(p^{2}+m^{2}\right)^{\frac{1}{4}}}\right\|_{q} \\
\leq\left\|\left(\frac{1}{|p|^{\frac{1}{2}}}-\frac{|p|}{\left(p^{2}+m^{2}\right)^{\frac{3}{4}}}\right)|A|^{\frac{1}{2}}\right\|_{p}\left\||A|^{\frac{1}{2}} \frac{1}{\left(p^{2}+m^{2}\right)^{\frac{1}{4}}}\right\|_{p},
\end{aligned}
$$

where $p=2 q>4$ and the unitary $A /|A|$ has been dropped from the second norm in (31). Then

$$
K_{1}=\left(\frac{1}{|p|^{\frac{1}{2}}}-\frac{|p|}{\left(p^{2}+m^{2}\right)^{\frac{3}{4}}}\right)|A|^{\frac{1}{2}},
$$

is a compact operator in $\mathcal{C}_{n}, n>2$ by Lemma 2.1 of Seiler and Simon 22 on integral operators of the form $f(p) g(x)$ and, by the same lemma as stated in Ref. 21 .

$$
\left\|K_{1}\right\|_{C_{n}} \leq(2 \pi)^{-\frac{2}{n}}\left\|\frac{1}{|p|^{\frac{1}{2}}}-\frac{|p|}{\left(p^{2}+m^{2}\right)^{\frac{3}{4}}}\right\|_{L^{n}}\left\||A|^{\frac{1}{2}}\right\|_{L^{n}} .
$$

Simple estimates give $\lim _{m=0}\left\|K_{1}\right\|_{4-\epsilon}=0$ for $0<\epsilon<2$, and since $\left\|K_{1}\right\|_{4-\epsilon} \geq$ $\left\|K_{1}\right\|_{4+\epsilon}$ is a general property of $\mathcal{C}_{n}$ spaces,

$$
\lim _{m=0}\left\|K_{1}\right\|_{4+\epsilon}=0
$$


Referring again to (31), let

$$
K_{2}(m)=|A|^{\frac{1}{2}} \frac{1}{\left(p^{2}+m^{2}\right)^{\frac{1}{4}}},
$$

where $K_{2} \in \mathcal{C}_{n}, n>4$ by Lemma 2.1 of Ref. 22 . At $m=0, K_{2}(0)=|A|^{\frac{1}{2}}|p|^{-\frac{1}{2}}$ and by writing $K_{2}(0)$ as the sum

$$
|A|^{\frac{1}{2}} \frac{1}{(m+|p|)^{\frac{1}{2}}}+|A|^{\frac{1}{2}}\left(\frac{1}{|p|^{\frac{1}{2}}}-\frac{1}{(m+|p|)^{\frac{1}{2}}}\right),
$$

each of the two operators belongs to $\mathcal{C}_{n}$ by Lemma 2.1, and hence so does $K_{2}(0) .24$ More simple estimates give

$$
\lim _{m=0}\left\|K_{2}(m)\right\|_{4+\epsilon} \leq\left\|K_{2}(0)\right\|_{4+\epsilon},
$$

where $0<\epsilon<2$. This together with (34) and (31) give

$$
\lim _{m=0}\left\|\frac{1}{|p|^{\frac{1}{2}}} A \frac{1}{\left(p^{2}+m^{2}\right)^{\frac{1}{4}}}-\frac{|p|}{\left(p^{2}+m^{2}\right)^{\frac{3}{4}}} A \frac{1}{\left(p^{2}+m^{2}\right)^{\frac{1}{4}}}\right\|_{q}=0
$$

for $q>2$, and hence the first norm on the right-hand side of (30) vanishes in the $m=0$ limit.

Repetition of analysis of this kind establishes that the remaining two norms in (30) also vanish at $m=0$, demonstrating (24) when $\Phi=0$.

\section{Further Work}

A major barrier to a better understanding of fermion determinants beyond two dimensions is the lack of criteria for counting zero modes. In three dimensions nothing is known, and in four dimensions only the difference of positive and negative chirality zero modes is known for a given background field. It is sometimes assumed that they all have one or the other chirality. A counterexample would settle this matter. In three dimensions the discovery of a topological invariant that counts zero modes would be of considerable importance to physics as well as mathematics.

\section{References}

1. E. Seiler, Commun. Math. Phys. 42 (1975) 163.

2. J. Schwinger, Phys. Rev. D82 (1951) 664.

3. M. P. Fry, Phys. Rev. D53 (1996) 980.

4. E. Seiler, Gauge Theories as a Problem of Constructive Quantum Field Theory and Statistical Mechanics, Lecture Notes in Physics Vol. 159 (Springer, Berlin/Heidelberg/New York, 1982). 
5. E. Seiler, in Gauge Theories: Fundamental Interactions and Rigorous Results, Proceedings of the International Summer School of Theoretical Physics, Poiana Brasov, Romania, 1981, edited by P. Dita, V. Georgescu, and R. Purice, Progress in Physics Vol. 5 (Birkhäuser, Boston, 1982), p. 263.

6. D. Brydges, J. Frölich and E. Seiler, Ann. Phys. (N.Y.) 121 (1979) 227.

7. D. Weingarten, Ann. Phys. (N.Y.) 126 (1980) 154.

8. D. H. Weingarten and J. L. Challifour, Ann. Phys. (N.Y.) 123 (1979) 61.

9. M. P. Fry, J. Math. Phys. 41 (2000) 1691.

10. Y. Aharonov and A. Casher, Phys. Rev. A19 (1979) 2461.

11. M. P. Fry, Phys. Rev. D62 (2000) 125007.

12. J. Schwinger, Phys. Rev. 128 (1962) 2425.

13. M. P. Fry, Phys. Rev. D54 (1996) 6444.

14. S. Deser, R. Jackiw and S. Templeton, Ann. Phys. (N.Y.) 140 (1982) 372; J Frölich and T. Kerler, Nucl Phys. B354 (1991) 369.

15. I. Aitchison and C. Fraser, Phys. Rev. D31 (1985) 2605; H. W. Lee, P. Y. Pac and H. K. Shin, Phys. Rev. D40 (1989) 4202; D. Cangemi, E. D'Hoker and G. Dunne, Phys. Rev. D51 (1995) R2513; V. P. Gusynin and I. A. Shovkovy, Can. J. Phys. 74 (1996) 282; J. Math. Phys. 40 (1999) 5406.

16. G. V. Dunne and T. M. Hall, Phys. Rev. D60 (1999) 065002.

17. H. Hogreve, R. Schrader and R. Seiler, Nucl. Phys. B142 (1978) 525.

18. C. Vafa and E. Witten, Nucl. Phys. B234 (1984) 173.

19. N. Dunford and J. T. Schwartz, Linear Operators, Part II (Interscience, New York, 1963).

20. B. Simon, Adv. Math. 24 (1977) 244.

21. B. Simon, Trace Ideals and their Applications, London Mathematical Society Lecture Note Series 35 (Cambridge University Press, Cambridge, England, 1979).

22. E. Seiler and B. Simon, Commun. Math. Phys. 45 (1975) 99.

23. E. Seiler, Phys. Rev. D22 (1980) 2412.

24. E. Seiler (private communication). 